BULL. AUSTRAL. MATH. SOC.

VOL. $26(1982), 29-37$.

\title{
WEIGHT FUNCTIONS WHICH ADMIT \\ TCHEBYCHEFF QUADRATURE
}

\section{Franz Peherstorfer}

\begin{abstract}
We describe a class of weight functions, which admit Tchebycheff quadrature.
\end{abstract}

Let integers $n, m \in N_{0}, m \leq 2 n-1$, and a nonnegative continuous weight function $w$ on $(-1,+1)$ be given. We call a quadrature formula of the type

$$
\int_{-1}^{+1} f(x) w(x) d x=\sum_{i=1}^{n} \lambda_{i} f\left(x_{i}\right)+R_{n}(f)
$$

a $(2 n-1-m, n, w)$ Tchebycheff quadrature formula on $[-1,+1]$, if the following three conditions are fuifilled: $-1<x_{1}<x_{2}<\ldots<x_{n}<1$, $\lambda_{1}=\lambda_{2}=\ldots=\lambda_{n}=1 / n$, and $R_{n}(f)=0$ for all $f \in \mathbb{P}_{2 n-1-m}$, where $\mathbb{P}_{2 n-1-m}$ is the set of polynomials of degree at most $2 n-1-m$. We say that the weight function $w$ admits $T$-quadrature, if for each $n \in N$ there exist nodes $x_{1}, \ldots, x_{n}$, such that (1) is a $(n, n, w)$ Tchebycheff quadrature formula.

UIIman [5] has shown that the weight function

$$
w_{\alpha}(x)=\frac{1}{\pi \sqrt{1-x^{2}}} \frac{1+\alpha x}{1+\alpha^{2}+2 \alpha x}, x \in(-1,+1), \alpha \in\left[-\frac{1}{2}, \frac{3}{2}\right],
$$

admits T-quadrature. So far these are the only known weight functions

Received 2 March 1982. 
with this property (compare [1]). In this paper we describe a class of weight functions, which admit T-quadrature.

We denote the closure and the boundary of a set $E \subset \mathbb{C}$ by $\bar{E}$ and $\partial E$ respectively. The open disk of radius $r \quad\{z \in \mathbf{C}|| z \mid<r\}$ is denoted by $U_{p}$. As usual the index $r$ is omitted for $r=1$. Furthermore $T_{k}$, $k \in N_{0}$, denotes the Tchebycheff polynomial of first kind of degree $k$.

For the following theorem see also Theorem 1 of [4].

THEOREM 1. The following three properties are equivalent:

(a) a quadrature formula based on nodes $x_{1}, \ldots, x_{n} \in \mathbf{R}$, $-1<x_{1}<x_{2}<\ldots<x_{n}<1$, is a $(2 n-1-m, n, w)$

Tchebycheff quadrature formula on $[-1,+1]$;

(b) $1-\frac{z t_{2 n}^{\prime}(z)}{n t_{2 n}(z)}=1+\sum_{k=1}^{2 n-1-m} c_{k} z^{k}+o\left(z^{2 n-m}\right)$ for $z \in U$, where $t_{2 n}(z)=\prod_{j=1}^{n}\left(z^{2}-2 \cos \varphi_{j} z+1\right), \varphi_{j}=\arccos x_{j}$, and
$c_{k}=2 \int_{-1}^{+1} T_{k}(x) \omega(x) d x$ for $k=1, \ldots, 2 n-1-m ;$

(c) $t_{2 n}(z)=\exp \left(n \int_{0}^{z} \frac{1-F(\xi)}{\xi} d \xi\right)+o\left(z^{2 n-m}\right)$ for $z \in U$, where $F(\xi)=1+\sum_{k=1}^{\infty} c_{k} \xi^{k}$ and $c_{k}=2 \int_{-1}^{+1} T_{k}(x) w(x) d x$ for $k \in N$.

Proof. $(a) \Rightarrow(b)$. Since $c_{k}=2 \int_{-1}^{+1} T_{k}(x) \omega(x) d x=\frac{2}{n} \sum_{j=1}^{n} \cos k \varphi_{j}$ for $k=0, \ldots, 2 n-1-m$, we deduce that

$$
\frac{1}{n} \sum_{j=1}^{n}\left(\frac{1}{1-e^{i \varphi_{j}}}+\frac{1}{1-e^{-i \varphi_{j}} j_{z}}-1\right)=1+\sum_{k=1}^{2 n-1-m} c_{k} z^{k}+o\left(z^{2 n-m}\right) \text { for } z \in U .
$$

Using the fact that 
(2)

$$
\frac{1}{n} \sum_{j=1}^{n}\left(\frac{1}{1-e^{i \varphi_{j}}}+\frac{1}{1-e^{-i \varphi_{j}}}-1\right)=1-\frac{z t_{2 n}^{\prime}(z)}{n t_{2 n}(z)}
$$

the implication is proved.

$(b) \Rightarrow(c)$. Taking into consideration the facts that $t_{2 n}$ has all zeros on the circumference and that $t_{2 n}(0)=1$, we obtain

$$
\frac{1}{n} \ln t_{2 n}(z)=\int_{0}^{z} \frac{1-F(\xi)}{\xi} d \xi+O\left(z^{2 n-m}\right)
$$

from which the assertion follows.

(c) $\Rightarrow(a)$. Since

$$
1-\frac{z t_{2 n}^{\prime}(z)}{n t_{2 n}(z)}=1+\sum_{k=1}^{2 n-1-m} c_{k} z^{k}+o\left(z^{2 n-m}\right)
$$

we get with the aid of (2) that

$$
c_{k}=\frac{2}{n} \sum_{j=1}^{n} \cos k \varphi_{j} \text { for } k=0, \ldots, 2 n-1-m .
$$

Henceforth we denote by

$$
q_{n}^{*}(z)=z^{n} \bar{q}\left(z^{-1}\right)=\bar{\gamma} \prod_{i=1}^{n}\left(1-\bar{z}_{i} z\right)
$$

the reciprocal polynomial of

$$
q_{n}(z)=\gamma \prod_{i=1}^{n}\left(z-z_{i}\right), \gamma, z, z_{i} \in \mathbf{C} .
$$

As a consequence of Theorem 1 we obtain (compare also [2, Theorem 1]) COROLLARY 1. Let

$$
F(z)=1+\sum_{k=1}^{\infty} c_{k} z^{k}
$$

where

$$
c_{k}=2 \int_{-1}^{+1} T_{k}(x) w(x) d x
$$


for $k \in \mathbf{N}$. Further let $p_{n}^{*}$ be defined by

$$
p_{n}^{*}(z)=\exp \left(n \int_{0}^{z} \frac{1-F(\xi)}{\xi} d \xi\right)+o\left(z^{n+1}\right)
$$

and assume that $p_{n}$ has all zeros in $\bar{U}_{\frac{1}{2}}$. The quadrature formula based on nodes which are the zeros of the polynomial

$$
q_{n}(x)=\operatorname{Re}\left\{p_{n}\left(e^{i \varphi}\right)-\left(p_{n}(0) / 2\right)\right\}, \quad x=\cos \varphi, \varphi \in[0, \pi],
$$

is a $(n, n, w)$ Tchebycheff quadrature formula on $[-1,+1]$.

Proof. Since $p_{n}$ has all zeros in $\bar{U}_{\frac{1}{2}}$, it follows from $[3, p .80]$ that all zeros of $p_{n}-p_{n}(0) / 2$ lie in the circle

$$
|z| \leq \frac{1}{2}+\left|p_{n}(0) / 2\right|^{1 / n}<1 .
$$

Thus the cosine polynomial $\operatorname{Re}\left\{p_{n}\left(e^{i \varphi}\right)-\left(p_{n}(0) / 2\right)\right\}$ has $n$ simple zeros in $(0, \pi)$. Setting

$$
t_{2 n}(z)=p_{n}^{*}(z)+z^{n}\left(p_{n}(z)-p_{n}(0)\right)
$$

the assertion follows from Theorem $1(c)$ and the fact that

$$
z^{-n} t_{2 n}(z) / 2=\operatorname{Re}\left\{p_{n}(z)-\left(p_{n}(0) / 2\right)\right\} \text { for } z=e^{i \varphi}, \varphi \in[0, \pi] \text {. }
$$

The following corollary gives us an extension of some results of UIIman [5] and Geronimus [2].

COROLLARY 2. Let $z \in N$ be fixed and let

$$
p_{i}(z)=\prod_{i=1}^{i}\left(z-\alpha_{i}\right) \text {, }
$$

$\alpha_{i} \in \bar{U}_{\frac{1}{2}}$ real or complex conjugate. Put

$$
w(x)=\frac{1}{\pi \sqrt{1-x^{2}}} \frac{1}{2} \sum_{i=1}^{l} \frac{1+\alpha_{i} x}{1+\alpha_{i}^{2}+2 \alpha_{i} \cdot x} \text { for } x \in(-1,+1)
$$

The quadrature formula based on nodes which are the zeros of the polynomial $(n \in \mathbb{N})$ 


$$
q_{\ln }(x)=\operatorname{Re}\left\{\left(p_{Z}\left(e^{i \varphi}\right)\right)^{n}-\left(\left(p_{\eta}(0)\right)^{n} / 2\right)\right\}, \quad x=\cos \varphi, \varphi \in[0 ; \pi],
$$

is a $\left(l_{n}, l_{n}, w\right)$ Tchebycheff quadrature formula on $[-1,+1]$.

Proof. Using the notation of Corollary 1 we obtain $\left(z=e^{i \varphi}\right.$, $\varphi \in[0, \pi])$

$$
\pi w(\cos \varphi) \sin \varphi=\operatorname{Re}\left\{\frac{1}{2} \sum_{i=1}^{l} \frac{1}{1-\alpha_{i} z}\right\}=\operatorname{Re} F(z)
$$

Hence

$$
\exp \left(\ln \int_{0}^{z} \frac{1-F(\xi)}{\xi} d \xi\right)=\prod_{i=1}^{i}\left(1-\alpha_{i} z\right)^{n} .
$$

In view of Corollary 1 the assertion is proved.

LEMMA 1. Let $E_{1}, E_{2}, E, \bar{E}_{1} \subset \bar{E}_{2} \subset E, 0 \in E_{1}$, be bounded simple connected domains in the complex plane, such that

$$
F(z)=1+\sum_{k=1}^{\infty} c_{k} z^{k}
$$

is analytic on $E$ and

$$
\delta:=\left(\max _{z \in \partial E_{1}}|z|\right) /\left(\min _{\xi \in \partial E_{2}}|\xi|\right)<1 .
$$

Put $G(z)=\sum_{k=1}^{\infty}\left(c_{k} / k\right) z^{k}$. If

$$
-\frac{1}{n} \ln (-1+(1 / \delta))+\ln \delta \leq \min _{\xi \in \partial E_{2}} \operatorname{Re} G(\xi)-\max _{z \in \partial E_{1}} \operatorname{Re} G(z),
$$

then $p_{n}^{*}$, defined by $p_{n}^{*}(z)=\exp (-n G(z))+o\left(z^{n+1}\right)$, has no zero in $E_{1}$.

Proof. Put

$$
H(z)=\exp \left(\int_{0}^{z} \frac{1-F(\xi)}{\xi} d \xi\right)=\exp (-G(z)) \text { for } z \in E
$$

and assume that $p_{n}^{*}$ has a zero $v \in E_{1}$. Since 


$$
(H(z))^{n}=p_{n}^{*}(z)+\frac{1}{2 \pi i} \int_{\partial E_{2}}(z / \xi)^{n}\left(\frac{z}{\xi-z}\right)(H(\xi))^{n} \frac{d \xi}{\xi} \text { for } z \in E_{2}
$$

we obtain, by using the inequality

$$
\frac{1--\delta}{\delta} \leq|\xi / z|-1 \leq|(\xi / z)-1| \text { for } z \in \partial E_{1}, \quad \xi \in \partial E_{2},
$$

that

$$
\begin{aligned}
I & =\left|\frac{1}{2 \pi i} \int_{\partial E_{2}}(v / \xi)^{n}\left(\frac{v}{\xi-v}\right)\left(\frac{H(\xi)}{H(v)}\right]^{n} \frac{d \xi}{\xi}\right| \\
& \left.\left.<\max _{\xi \in \partial E_{2}}|H(\xi)|^{n}\right\} / \min _{z \in \partial E_{1}}|H(z)|^{n}\right\} \cdot \delta^{n} \cdot \frac{\delta}{(I-\delta)} .
\end{aligned}
$$

Taking into consideration the fact that

$$
|H(\xi)|=\exp (-\operatorname{Re} G(\xi))
$$

we get

$$
\max _{\xi \in \partial E_{2}}|H(\xi)|=1 /\left(\exp \left(\min _{\xi \in \partial E_{2}} \operatorname{Re} G(\xi)\right)\right),
$$

respectively

$$
\min _{z \in \partial E_{1}}|H(z)|=1 /\left(\exp \left(\max _{z \in \partial E_{1}} \operatorname{Re} G(z)\right)\right)
$$

Thus it follows that

$$
\begin{aligned}
1 & \left.\left.<\max _{\xi \in \partial E_{2}}|H(\xi)|\right) / \min _{z \in \partial E_{1}}|H(z)|\right) \cdot \delta \cdot\left(\frac{\delta}{1-\delta}\right)^{1 / n} \\
& \left.=\exp _{z \in \partial E_{1}} \operatorname{Re} G(z)-\min _{\xi \in \partial E_{2}} \operatorname{Re} G(\xi)\right) \cdot \delta \cdot\left(\frac{\delta}{1-\delta}\right)^{1 / n} \leq 1
\end{aligned}
$$

which is a contradiction.

THEOREM 2. (a) Let $\sigma$ be an odd bounded nondecreasing function on $[-\pi, \pi]$, such that $\int_{-\pi}^{+\pi} d \sigma(t)=1$. Suppose that $\alpha \in[-1 / 8,1 / 8]$. Then 


$$
\omega(x)=\frac{1}{\pi \sqrt{1-x^{2}}} \operatorname{Re} \int_{-\pi}^{+\pi} \frac{d \sigma(t)}{1-\alpha e^{i t} z}, \quad x=\frac{1}{2}\left(z+\frac{1}{z}\right), \quad z=e^{i \varphi}, \varphi \in(0, \pi),
$$

admits T-quadrature on $[-1,+1]$.

(b) Let $\Phi(z)=a_{0}+a_{1} z+a_{2} z^{2}+\ldots, a_{i} \in \mathbf{R}$ for $i \in \mathbf{N}_{0}$, be analytic on $U_{8}$ and suppose that $|\Phi(z)| \leq 1 / 8$ for $z \in U_{8}$. Then

$$
w(x)=\frac{1}{\pi \sqrt{1-x^{2}}} \operatorname{Re} \frac{1}{1+z \Phi(z)}, x=\frac{1}{2}\left(z+\frac{1}{z}\right), \quad z=e^{i \varphi}, \varphi \in(0, \pi)
$$

admits T-quadrature on $[-1,+1]$.

Proof. (a) Let

$$
F(z)=\int_{-\pi}^{+\pi} \frac{d \sigma(t)}{1-\alpha e^{i t} z}=1+\sum_{k=1}^{\infty} c_{k} z^{k} .
$$

Then $F$ is analytic on $U_{1 /|\alpha|}$ and

$$
\operatorname{Re} F(z)=\int_{-\pi}^{+\pi} \operatorname{Re} \frac{1}{1-\alpha e^{i t} z} d \sigma(t) \geq \frac{1}{1+|\alpha|} \text { for }|z| \leq 1 \text {. }
$$

Thus $\pi \sqrt{1-x^{2}} \omega(x)=\operatorname{Re} F\left(e^{i \varphi}\right), x=\cos \varphi, \varphi \in(0, \pi)$, is positive and continuous on $(-1,+1)$. Furthermore we have that

$$
c_{k}=\frac{2}{\pi} \int_{0}^{\pi} \operatorname{Re} F\left(e^{i \varphi}\right) e^{-i n \varphi} d \varphi=2 \int_{-1}^{+1} T_{k}(x) \omega(x) d x
$$

In view of Corollary 1 it suffices to show that $p_{n}^{*}$ has no zero in $U_{2}$. Putting $E_{1}=U_{2}$ and $E_{2}=U_{4}$ in Lemma 1 and observing that

$$
G(z)=-\int_{-\pi}^{+\pi} \ln \left(1-\alpha e^{i t} z\right) d \sigma(t)
$$

we obtain, by using the inequality

$$
1-|\alpha| r \leq\left|1-\alpha e^{i t} z\right| \leq 1+|\alpha| r \text { for } z=r e^{i \varphi} \text {, }
$$

that 


$$
\begin{aligned}
\min _{\xi \in \partial U_{4}} \int_{-\pi}^{+\pi} \ln 1 /\left|1-\alpha e^{i t} \xi\right| d \sigma(t) & -\max _{z \in \partial U_{2}} \int_{-\pi}^{+\pi} \ln 1 /\left|1-\alpha e^{i t} z\right| d \sigma(t) \\
& \geq \ln 1 /(1+4|\alpha|)-\ln 1 /(1-2|\alpha|) \geq \ln \frac{\pi}{2}=\ln \delta
\end{aligned}
$$

from which the assertion follows.

(b) Let $F$ be defined as in the proof of (a). Then $F$ is analytic on $U_{8}$ and $\operatorname{Re} F(z)>\frac{1}{2}$ for $z \in U_{8}$. Thus $F(z)=1 /(1+z \Phi(z))$, where $\Phi$ is analytic on $U_{8}$ and satisfies $|\Phi(z)| \leq 1 / 8$ for $z \in U_{8}$.

EXAMPLE. From Theorem 2 we obtain immediately that the weight function

$$
w(x)=\frac{1}{\pi \sqrt{1-x^{2}}} \frac{1}{2} \sum_{i=1}^{2} \frac{1+\alpha_{i} x}{1+\alpha_{i}^{2}+2 \alpha_{i} x}, x \in(-1,+1),
$$

which was considered in Corollary 2 , admits $T$-quadrature on $[-1,+1]$, if $\left|\alpha_{i}\right| \leq 1 / 8$ for $i=1, \ldots, i$.

\section{References}

[1] Walter Gautschi, "Advances in Chebyshev quadrature", Numerical analysis, 100-12l (Proc. Dundee Conf. Numerical Analysis, 1975. Lecture Notes in Mathematics, 506. Springer-Verlag, Berlin, Heidelberg, New York, 1976).

[2] Я.Л. Геронимус [Ja.L. Geronimus], "О нвадратурной формуле Чеб̈ьшева", Izv. Akad. Nauk SSSR Ser. Mat. 33 (1969), 1182-1207. English Transl: "Čebyšev's quadrature formula", Math. USSR - Izv. 3 (1969), 1115-1138.

[3] Morris Marden, Geometry of polynomials (Mathematical Surveys, 3. American Mathematical Society, Providence, Rhode Island, 1966).

[4] Franz Peherstorfer, "Characterization of positive quadrature formulas", SIAM J. Math. Anal. 12 (1981), 935-942. 
[5] J.L. UlIman, "A class of weight functions that admit Tchebycheff quadrature", Michigan Math. J. 13 (1966), 417-423.

Johannes Kepler Universitat Linz,

Institut für Mathematik,

Alten berger Strasse 69 ,

A-4045 Linz,

Austria. 